\title{
Multiple scales of diversification within natural populations of archaea in hydrothermal chimney
}

\section{2 biofilms}

\section{William J. Brazelton $^{1 *}$, Mitchell L. Sogin ${ }^{2}$, and John A. Baross ${ }^{1}$}

$4{ }^{1}$ School of Oceanography and Center for Astrobiology and Early Evolution, University of Washington

2 Josephine Bay Paul Center, Marine Biological Laboratory at Woods Hole

6

* corresponding author: Box 357940, School of Oceanography, University of Washington, Seattle, WA

8 98195. telephone: 206-221-5755. fax: 206-543-6073. braz@u.washington.edu

10 running title: Microdiversity within a single-species biofilm

\section{Abstract}

Corroborative data collected from 16S rRNA clone libraries, intergenic transcribed spacer (ITS) region

14 clone libraries, and 16S rRNA hypervariable region tag pyrosequencing demonstrate microdiversity within single-species archaeal biofilms of the Lost City Hydrothermal Field. Both 16S rRNA clone

16 libraries and pyrosequencing of the V6 hypervariable region show that Lost City Methanosarcinales (LCMS) biofilms are dominated by a single sequence, but the pyrosequencing dataset also reveals the presence of an additional 1654 rare sequences. Clone libraries constructed with DNA spanning the V6 hypervariable region and ITS show that multiple ITS sequences are associated with the same dominant

20 V6 sequence. Furthermore, ITS variability differed among three chimney samples, and the sample with the highest ITS diversity also contained the highest V6 diversity as measured by clone libraries as well

22 as tag pyrosequencing. These results indicate that the extensive microdiversity detected in V6 tag sequences is an underestimate of genetic diversity within the archaeal biofilms. 


\section{INTRODUCTION}

26 Biofilms coating carbonate chimneys of the Lost City Hydrothermal Field (Kelley et al., 2005) are dominated by a single 16S rRNA phylotype referred to as Lost City Methanosarcinales (LCMS;

28 Schrenk et al., 2004; Brazelton et al., 2006). Previous studies have shown that $>80 \%$ of all cells in carbonate chimneys venting $20-90^{\circ} \mathrm{C}, \mathrm{pH}$ 9-11 fluids hybridize to a fluorescent in situ hybridization

30 (FISH) probe specific to LCMS (Schrenk et al., 2004). LCMS has resisted laboratory cultivation, but it is presumed to subsist on the high concentrations of hydrogen and/or methane gas venting from the

32 carbonate chimneys (Kelley et al., 2005).

34 Previous studies have shown ecologically relevant genetic and physiological diversity within natural populations of archaea and bacteria that initially seemed to contain very little genetic diversity based on

36 16S rRNA sequences. For example, bacterioplankton with $>99 \%$ similar 16S rRNA sequences can harbor extensive genomic variation (Thompson et al., 2005) and comprise many ecologically distinct

38 strains (Hunt et al., 2008). Variation in the intergenic transcribed spacer (ITS) region, which is less conserved than $16 \mathrm{~S}$ rRNA, is often a better predictor of genomic and ecological variation. ITS sequence

40 variation delineates cyanobacterial 'ecotypes' that have substantial differences in genomic content (Rocap et al., 2003) and physiological differences linked to distinct localizations within water columns

42 (West et al., 2001) or microbial mats (Ferris et al., 2003). Environmental sequencing of the ITS region has also proved useful in resolving genetically distinct clusters within uncultivated organisms belonging

44 to the Thermococcales group of thermophilic archaea (Huber et al., 2006), the Group I Crenarchaeota (Schleper et al., 1998, Nicol et al., 2006), and the SAR11 group of marine bacteria (Garcia-Martinez \&

46 Rodriguez-Valera, 2000).

In this paper we test whether the LCMS phylotype consists of genetically distinct subpopulations by 
thoroughly exploring the sequence diversity in the 16S rRNA gene as well as the ITS region, utilizing

50 both Sanger sequencing of clone libraries and tag pyrosequencing of the V6 hypervariable region.

\section{RESULTS AND DISCUSSION}

\section{$16 S$ rRNA clone library}

54 An archaeal 16S rRNA clone library was constructed (by the DOE Joint Genome Institute) from a single carbonate chimney collected from the main chimney structure at Lost City known as Poseidon (sample

56 LC0424). Sequences were obtained from 486 clones (GenBank accession numbers FJ791302FJ791787), all of which showed high sequence similarity to the previously published (Schrenk et al., 2004) 16S rRNA sequence of LCMS. After screening for length and quality, 200 clone sequences each containing at least $1250 \mathrm{bp}$ were selected for further analysis.

All 200 clones were at least $98.8 \%$ similar over the 1253 bp alignment, but 163 unique sequences were

62 detected (Figure 1). Although the evenness of unique sequences is high (Table 1) because the most common sequence was shared by only 36 clones, no other sequence was shared by more than two

64 clones. Most of the variations among sequences were substitutions; insertions and deletions were comparatively rare (Table 1). Similar results are achieved if only the V6 hypervariable region is

66 considered (where the V6 is defined by the primers used for V6 tag pyrosequencing described below). Of the 200 clones, 179 have identical V6 sequences, and the 21 variant clones represent 19 additional 68 sequences.

70 Comparing the variant sequences to the most common sequence yields a mutation rate of $0.15 \%$ for the nearly full-length gene and $0.16 \%$ for the V6 region. Because the sequence differences are rare and

72 mostly unique, it is possible that they could be caused by DNA polymerase error. A Taq DNA 
polymerase error rate of $2.3 \times 10^{-5}$ per base per cycle (Li et al., 2006), however, would only contribute

$740.046 \%$ sequence variation after 20 cycles of amplification (JGI Standard Protocol) during the polymerase chain reaction. Therefore, polymerase error is unlikely to account for all of the diversity

76 observed in our clone libraries.

$78 \quad$ V6 hypervariable region tag sequences

We obtained 16,260 tag sequences of the V6 hypervariable region of the archaeal 16S rRNA gene from another sample (LC1408) of the same chimney used for the 16S rRNA clone library. More than $91 \%$ of these tags were assigned to the family Methanosarcinaceae by GAST (Huse et al. 2008) and showed an

82 extremely uneven abundance distribution. Of the 14,869 Methanosarcinaceae tags, $75 \%$ were identical to the corresponding V6 region of 179 of the 200 full-length $16 \mathrm{~S}$ rRNA clones. The remaining $25 \%$

84 (3695 tags) comprised 622 different sequences clustering into 235 operational taxonomic units (OTUs) at $97 \%$ sequence similarity (Figure 1).

The second most common V6 tag sequence (representing $5 \%$ of all tags) differs from the dominant 88 sequence by lacking the final GAG at the 3' end. The deletion was not caused by premature truncation of pyrosequencing extension because in each case the distal primer was accurately sequenced. The

90 sequence GAGAG at the 3' end of the V6 region is highly conserved in archaeal rRNA, but $0.8 \%$ of archaeal sequences, including many methanogens, in the RefHVR_v6 database (http://vamps.mbl.edu)

92 lack the final GAG (S. Huse, personal communication). Because this database is derived from traditional Sanger sequencing of clones, the GAG deletion in our data is unlikely to be caused by pyrosequencing

94 error. The lack of this deletion in our clone libraries, however, is puzzling.

96 Two additional samples (LC1404 and LC1443) collected from a different chimney showed very similar 
distributions, being dominated by the same sequence with a large diversity of very rare sequences

98 (Figure 2a). The temperature and fluid chemistry at this chimney was similar to the chimney from which sample LC1408 was collected, although samples LC1404 and LC1443 had much higher cell densities

100 (Table S1). The three samples together contained 72,577 tags assigned to the family Methanosarcinaceae representing 1654 different sequences and 536 operational taxonomic units at $97 \%$

102 sequence similarity. The extreme rarity of the diverse sequences raises questions regarding the effect of pyrosequencing error. Tag abundances decreased substantially with increasing distance from the most

104 dominant sequence, a trend that is consistent with the expected effect of random sequencing error from one dominant template. Some sequences, however, appeared much more frequently than others with the

106 same number of substitutions and indels (prominent peaks in Figure 2a), so these may represent genuine diversity above a background error rate.

Three additional features of our data argue against a significant contribution from pyrosequencing error

110 to the observed diversity. Firstly, the amount of sequence variation was too high to be generated by pyrosequencing error alone. Comparing all variant V6 tag sequences to the one dominant sequence

112 yielded mutation rates of $0.55-0.71 \%$ for the three samples (Table 1 ), while the error rate associated with the pyrosequencing technique and quality-filtering procedure used in this study should not exceed $0.16 \%$

114 (Huse et al. 2007). Most of the mutations were insertions and deletions, whose pyrosequencingassociated rates can vary depending on the template sequence, but the substitution rates $(0.15-0.20 \%)$

116 were also much higher than the maximum expected from pyrosequencing error $(0.03 \%$, Huse et al., 2007).

Secondly, many of the bases with the highest substitution rate in the V6 tags were also the most variable 120 bases in the clone library sequences. Positions outlined with a black box in Figure $2 \mathrm{~b}$ were the site of at 
least two substitutions in clone libraries (including the full-length library described above and the three

122 V6-ITS libraries described below). All of these positions also had greater than average substitution rates in the V6 tag dataset (indicated by orange and red shading in Figure 2b). It is highly unlikely that error

124 introduced by both Sanger sequencing of clone libraries and tag pyrosequencing could cause this correspondence in site-specific substitution rates. Furthermore, the transition/transversion ratios

126 associated with substitutions in the V6 tags were very similar to that found in the full-length clone libraries (Table 1).

Finally, pyrosequencing error alone cannot account for the high similarity between the V6 tag

130 distributions of the two samples from the same chimney (LC1404 and LC1443) compared to that of LC1408, which was collected from a different chimney. Although all three samples are very similar in

132 their complement of abundant sequences (Figure 2a), only a small proportion of the total sequences were shared among samples (Jaccard similarities of 22-26\%) due to the large number of rare sequences.

134 Interestingly, LC1404 and LC1443 both contained fewer unique sequences than sample LC1408 (Table 1), and the Bray-Curtis community similarity between the two samples from the same chimney was

136 higher than the community similarity between samples from different chimneys (see Supplementary Information for details). Although this comparison involves only three samples and thus is not strong

138 statistical evidence, it is suggestive that small differences in rare V6 tag sequences reflect environmental variation.

\section{V6-ITS clone libraries}

142 To directly compare the diversity of the V6 region within the LCMS biofilms to a marker known to be more variable in other organisms (Rocap et al., 2002), we constructed clone libraries of 1071 bp DNA

144 fragments spanning the 3' end of the 16S rRNA gene including the V6 hypervariable region and the 
intergenic transcribed spacer (ITS) region between the 16S and 23S rRNA genes. Approximately 150-

146200 clones were sequenced from each of the same three carbonate chimney samples used for V6 tag pyrosequencing. As expected, nearly all 197 V6-ITS clones from sample LC1408 shared the same V6

148 sequence that dominated the pyrosequencing dataset. Only 7 clones had variant V6 sequences (Figure 1), and each of these were unique and the result of transitions. V6-ITS clones from samples LC1404 and

150 LC1443 were also dominated by a single V6 sequence with only a few variants mostly caused by transitions. Sequencing error cannot be discounted as a source for such a small number of V6 variants.

Although the ITS regions of all 516 V6-ITS clones were of nearly identical size (360 bp) and >98\%

154 similar to each other, 104 different sequences were detected among the three samples. For samples LC1408 and LC1443 the mutation rate within the ITS region (0.24\% and $0.16 \%)$ was higher than the

156 mutation rate within the V6 hypervariable region ( $0.06 \%$ and $0.11 \%)$, but in sample LC1404 the ITS region exhibited even less variation $(0.02 \%)$ than in the V6 $(0.04 \%)$ (Table 1$)$. The variation in the V6

158 regions of the V6-ITS clones were substantially lower than that observed for the V6 region of the $16 \mathrm{~S}$ rRNA clones, even though 34-38 cycles were required for amplification of the V6-ITS clones, compared

160 to 20 cycles for the $16 \mathrm{~S}$ rRNA clones. We conclude that error introduced during amplification and cloning does not appear to greatly affect the observed trends in ITS sequence variation.

The ITS region of LCMS encodes an Ala-tRNA and shows sequence homology with the ITS regions of

164 several methanogens (Figure S1). Sequence variations were most commonly associated with two predicted stem-loop structures in the region upstream of the tRNA gene (Figure 3a). The five most

166 common variations were present in 10-30 clones per library; most positions were variable in only 0-1 clones (Figure 3a). The highly non-random distribution of sequence variation along the length of the ITS 168 argues strongly against a large contribution of variation from sequencing error. 
170 Sample LC1408 contained 47 different ITS sequences (Figure 1), more than LC1404 (23 sequences) or LC1443 (43 sequences). The evenness of sample LC1408 was higher than that of the other samples

172 (Table 1), as the most common sequence comprised just $37.8 \%$ of all clones. The greater evenness in LC1408 ITS sequences may be due, in part, to the higher number of cycles required for sufficient PCR

174 amplification of this sample, but this effect is not expected to be large for reasons described above and in the Supplementary Information. Furthermore, it is intriguing that the ITS clone libraries as well as the

176 V6 tag datasets showed the highest diversity and evenness in sample LC1408 and the least diversity and evenness in sample LC1404 (Table 1). This correspondence between genetic markers and sequencing

178 technologies supports the observed trends as reliable indicators of biological diversity and not artifacts of the methodology.

180

The ITS region appears to reveal a scale of diversity that is not reflected in 16S rRNA sequences.

182 Compared to the 16S rRNA clone libraries and V6 tag pyrosequencing datasets, the ITS clones showed a more even abundance distribution of sequences (as shown in the higher evenness values in Table 1 and

184 in Figure S2). Of all 516 V6-ITS clones, 231 contained ITS sequence variations, and eight of these variants occurred more than twice. In contrast, none of the $16 \mathrm{~S}$ rRNA variants occurred more than twice,

186 so it is possible that many of these variants were generated by sequencing error. Of the 231 clones with variant ITS sequences, 221 clones had identical V6 sequences. The 10 exceptions involved 9 different

188 V6 sequences and 6 different ITS sequences. Thus nearly all of the observed ITS variation is associated with the same dominant V6 sequence, and it is likely that a tag pyrosequencing study of the LCMS

190 biofilm with primers targeting the ITS region would reveal even more microdiversity than the thousands of V6 sequence types found in this study.

192 
Multiple studies have shown that large genomic differences are possible among organisms with only

194 small variations in 16S rRNA sequence (Beja et al., 2002; Welch et al., 2002; Rocap et al., 2003;

Thompson et al., 2005), but further work is necessary to determine if the microdiversity reported in our

196 study is associated with larger scale genomic variations leading to important physiological and ecological consequences. The V6 tag dataset alone does not compel rejection of a null hypothesis of

198 ecologically-neutral genetic drift within a clonal population because it is possible for the many extremely rare V6 tag sequences to reflect 'background' mutations not yet affected by selection and

200 speciation. The highly non-random nature of the ITS variation, however, provides stronger evidence for ecologically relevant diversity. The markedly different distributions of ITS genotypes among chimney

202 samples (Figure 3b) may be an indication that the biofilm community contains several distinct subpopulations represented by different ITS genotypes. Determining whether these subpopulations 204 represent physiologically and ecologically distinct units (i.e. ecotypes or species) will require further genomic and physiological experiments. In particular, these experiments should test the hypothesis that

206 differentiation within this one group of archaea is the result of subpopulations colonizing multiple niches within the chimney to maximize utilization of resources that are unavailable to other organisms due to 208 the extreme conditions of Lost City chimneys (Kelley et al., 2005; Brazelton et al., 2006).

210 The detection of so many rare V6 sequences was only technically feasible in this study due to the extremely low diversity of the Lost City carbonate chimneys. As sequencing technology continues to

212 improve in sensitivity, fidelity, and read length, measurements of even finer scale microdiversity and comparisons of variation across multiple genomic markers will become possible for systems with

214 greater diversity. This near-future technology could be used to test whether the rare microdiversity reported here is a natural feature of microbial populations or an unusual characteristic unique to this 216 extremophilic archaeal community. 


\section{Acknowledgements}

We thank Deborah Kelley, chief scientist, and the scientific parties of the 2003 and 2005 Lost City

220 Expeditions as well as the crews of the R/V Atlantis, DSV Alvin, R/V Ronald H. Brown, and DSV

Hercules. We also appreciate technical assistance and helpful discussions with Ekaterina Andreishcheva,

222 Sue Huse, and Julie Huber. This research was supported by the W.M. Keck Foundation to MLS, the

NASA Astrobiology Institute through the Carnegie Institution for Science to JAB and through the MBL 224 to MLS.

\section{References}

Beja, O., Koonin, E.V., Aravind, L., Taylor, L.T., Seitz, H., Stein, J.L. et al. (2002) Comparative genomic analysis of archaeal genotypic variants in a single population and in two different oceanic provinces. Appl Environ Microbiol 68: 335-345.

Brazelton, W.J., Schrenk, M.O., Kelley, D.S., and Baross, J.A. (2006) Methane- and sulfur-metabolizing microbial communities dominate the Lost City hydrothermal field ecosystem. Appl Environ Microbiol 72: 6257-6270.

Ferris, M.J., Kuhl, M., Wieland, A., and Ward, D.M. (2003) Cyanobacterial ecotypes in different optical microenvironments of a 68 degrees $\mathrm{C}$ hot spring mat community revealed by $16 \mathrm{~S}-23 \mathrm{~S}$ rRNA internal transcribed spacer region variation. Appl Environ Microbiol 69: 2893-2898.

Garcia-Martinez, J., and Rodriguez-Valera, F. (2000) Microdiversity of uncultured marine prokaryotes: the SAR11 cluster and the marine Archaea of Group I. Mol Ecol 9: 935-948.

238 Huber, J.A., Butterfield, D.A., and Baross, J.A. (2006) Diversity and distribution of subseafloor Thermococcales populations in diffuse hydrothermal vents at an active deep-sea volcano in the northeast Pacific Ocean. J Geophys Res Biogeosciences 111: G04016. 
Hunt, D.E., David, L.A., Gevers, D., Preheim, S.P., Alm, E.J., and Polz, M.F. (2008) Resource

partitioning and sympatric differentiation among closely related bacterioplankton. Science 320: 1081-1085.

244 Huse, S.M., Huber, J.A., Morrison, H.G., Sogin, M.L., and Welch, D.M. (2007) Accuracy and quality of massively parallel DNA pyrosequencing. Genome Biol 8: R143.

246 Kelley, D.S., Karson, J.A., Fruh-Green, G.L., Yoerger, D.R., Shank, T.M., Butterfield, D.A. et al. (2005) A serpentinite-hosted ecosystem: the Lost City hydrothermal field. Science 307: 1428-1434.

Li, M., Diehl, F., Dressman, D., Vogelstein, B., and Kinzler, K.W. (2006) BEAMing up for detection and quantification of rare sequence variants. Nat Methods 3: 95-97.

Lowe, T.M. and Eddy, S.R. (1997) tRNAscan-SE: a program for improved detection of transfer RNA genes in genomic sequence. Nucleic Acids Res 25: 955-964.

252 Markham, N. R. and Zuker, M. (2005) DINAMelt web server for nucleic acid melting prediction. Nucleic Acids Res 33: W577-W581.

254 Nicol, G.W., Tscherko, D., Chang, L., Hammesfahr, U., and Prosser, J.I. (2006) Crenarchaeal community assembly and microdiversity in developing soils at two sites associated with deglaciation. Environ Microbiol 8: 1382-1393.

Rocap, G., Distel, D.L., Waterbury, J.B., and Chisholm, S.W. (2002) Resolution of Prochlorococcus and Synechococcus ecotypes by using 16S-23S ribosomal DNA internal transcribed spacer sequences. Appl Environ Microbiol 68: 1180-1191.

Rocap, G., Larimer, F.W., Lamerdin, J., Malfatti, S., Chain, P., Ahlgren, N.A. et al. (2003) Genome divergence in two Prochlorococcus ecotypes reflects oceanic niche differentiation. Nature 424: 1042-1047.

Schleper, C., DeLong, E.F., Preston, C.M., Feldman, R.A., Wu, K.Y., and Swanson, R.V. (1998) Genomic analysis reveals chromosomal variation in natural populations of the uncultured 
psychrophilic archaeon Cenarchaeum symbiosum. J Bacteriol 180: 5003-5009.

266 Schrenk, M.O., Kelley, D.S., Bolton, S.A., and Baross, J.A. (2004) Low archaeal diversity linked to subseafloor geochemical processes at the Lost City Hydrothermal Field, Mid-Atlantic Ridge. Environ Microbiol 6: 1086-1095.

Thompson, J.R., Pacocha, S., Pharino, C., Klepac-Ceraj, V., Hunt, D.E., Benoit, J. et al. (2005) Genotypic diversity within a natural coastal bacterioplankton population. Science 307: 13111313.

272 Welch, R.A., Burland, V., Plunkett, G.r., Redford, P., Roesch, P., Rasko, D. et al. (2002) Extensive mosaic structure revealed by the complete genome sequence of uropathogenic Escherichia coli. Proc Natl Acad Sci USA 99: 17020-17024.

West, N.J., Schonhuber, W.A., Fuller, N.J., Amann, R.I., Rippka, R., Post, A.F., and Scanlan, D.J. (2001) Closely related Prochlorococcus genotypes show remarkably different depth distributions in two oceanic regions as revealed by in situ hybridization using 16S rRNA-targeted oligonucleotides. Microbiology 147: 1731-1744.

\section{Figure Captions}

Figure 1. Comparison of tag pyrosequencing and clone library data from the same carbonate chimney.

282 All sequences from 200 nearly full-length 16S rRNA clones obtained from sample LC0424 were more than $97 \%$ similar to each other (A). Collection of 14,869 tag pyrosequences of the V6 hypervariable region from a different sample (LC1408) of the same chimney revealed much greater diversity (B). A clone library constructed with DNA from sample LC1408 spanning the V6 hypervariable region and the intergenic transcribed spacer (ITS) region showed more diversity in the ITS region (C).

Figure 2. Tag pyrosequences of the V6 hypervariable region reveal a wide range of highly similar, rare 
sequences. The relative abundance distribution (A) of 1654 different V6 sequences among the three

290 samples (LC1408, LC1404, LC1133) shows the extreme dominance of one sequence and the diversity of rare sequences. Differences (and similarities) among samples are more easily seen when the one

292 dominant sequence and sequences observed only once in the total dataset ('singletons') are omitted to show only the 483 most common variants (inset). Sequences are sorted along the $\mathrm{X}$ axis by distance to

294 the dominant sequence. The predicted secondary structure of the V6 region (B) was slightly modified from the archaeal structure on the Comparative RNA Web Site (http://www.rna.ccbb.utexas.edu) to fit

296 the dominant sequence. Those bases that are variable in at least two clones among all the clone libraries in this study (in boxes) are among the most highly variable (orange and red shading) in the V6 tag

298 dataset as well. All V6 sequence data is available at the VAMPS database, http://vamps.mbl.edu., under dataset name ICM_LCY_Av6 and in the NCBI Short Read Archive under submission number

300 SRP000912.

302 Figure 3. The variability of specific bases within the ITS region of Lost City Methanosarcinales differs among chimney samples. The secondary structure of the ITS (A) was predicted by UNAFOLD

304 (Markham and Zuker, 2005) and modified to match the tRNA structure predicted by tRNAscan-SE (Lowe and Eddy, 1997). Bases are color-coded to indicate the number of clones (out of 517 total) that

306 differed from the dominant sequence at that position, and the five most variable positions are numbered and compared among samples in (B). The most frequent variation, a $\mathrm{C}$ to $\mathrm{T}$ transition, occurred in 22

308 clones in sample LC1408, in 0 clones in LC1404, and 21 clones in LC1443. Accession numbers for clones including the V6 and ITS regions include GQ272945-GQ273460. 


\begin{tabular}{|c|c|c|c|c|c|c|c|c|c|c|}
\hline & $\underset{1}{\text { sample }}$ & $\begin{array}{c}\text { clones } \\
\text { or tags }\end{array}$ & $\begin{array}{c}\text { length } \\
\text { (bp) }\end{array}$ & $\begin{array}{c}\text { unique } \\
\text { sequen } \\
\text { ces }^{2}\end{array}$ & $\begin{array}{c}\text { total } \\
\text { mutati } \\
\text { on rate }\end{array}$ & $\begin{array}{c}\text { insertio } \\
\text { n rate }\end{array}$ & $\begin{array}{c}\text { deletion } \\
\text { rate }\end{array}$ & $\begin{array}{c}\text { substit } \\
\text { ution } \\
\text { rate }\end{array}$ & $\begin{array}{l}\mathrm{Ti} / \mathrm{Tv} \\
\text { ratio }\end{array}$ & evenness $^{4}$ \\
\hline \multirow[t]{2}{*}{$\begin{array}{c}\text { 16S } \\
\text { rRNA } \\
\text { clones }\end{array}$} & $\begin{array}{c}\text { LC0424 } \\
\text { (full } \\
\text { length) }\end{array}$ & 200 & 1253 & 163 & $0.15 \%$ & $0.01 \%$ & $0.01 \%$ & $0.13 \%$ & $\begin{array}{c}269 / 4 \\
5\end{array}$ & $\begin{array}{c}0.91 \pm \\
0.04\end{array}$ \\
\hline & $\begin{array}{c}\text { LC0424 } \\
\text { (V6 } \\
\text { region) }\end{array}$ & 200 & 65 & 20 & $0.16 \%$ & $0 \%$ & $0 \%$ & $0.16 \%$ & $18 / 3$ & $\begin{array}{c}0.21 \pm \\
0.07\end{array}$ \\
\hline \multirow{3}{*}{$\begin{array}{c}\text { V6 } \\
\text { pyrose } \\
\text { quenci } \\
\text { ng tags }\end{array}$} & LC1408 & 14,869 & 65 & 623 & $0.71 \%$ & $0.12 \%$ & $0.38 \%$ & $0.20 \%$ & 6.10 & $\begin{array}{c}0.267 \pm \\
0.007\end{array}$ \\
\hline & LC1404 & 32,340 & 65 & 472 & $0.55 \%$ & $0.16 \%$ & $0.24 \%$ & $0.16 \%$ & 4.47 & $\begin{array}{c}0.236 \pm \\
0.007\end{array}$ \\
\hline & LC1443 & 25,368 & 65 & 487 & $0.58 \%$ & $0.16 \%$ & $0.22 \%$ & $0.20 \%$ & 8.78 & $\begin{array}{c}0.247 \pm \\
0.007\end{array}$ \\
\hline & & & & 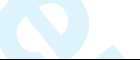 & & & & & & \\
\hline \multirow{3}{*}{$\begin{array}{c}\text { V6-ITS } \\
\text { clones } \\
\text { (V6 } \\
\text { region) }\end{array}$} & LC1408 & 196 & 65 & 8 & $0.06 \%$ & $0 \%$ & $0 \%$ & $0.06 \%$ & $8 / 0$ & $\begin{array}{c}0.11 \pm \\
0.06\end{array}$ \\
\hline & LC1404 & 132 & 65 & 4 & $0.04 \%$ & $0 \%$ & $0 \%$ & $0.04 \%$ & $6 / 0$ & $\begin{array}{c}0.09 \pm \\
0.09\end{array}$ \\
\hline & LC1443 & 189 & 65 & 12 & $0.11 \%$ & $0 \%$ & $0 \%$ & $0.11 \%$ & $12 / 1$ & $\begin{array}{c}0.16 \pm \\
0.07\end{array}$ \\
\hline \multirow{3}{*}{$\begin{array}{c}\text { V6-ITS } \\
\text { clones } \\
\text { (ITS } \\
\text { region) }\end{array}$} & LC1408 & 196 & 360 & 57 & $0.24 \%$ & $0.03 \%$ & $0.06 \%$ & $0.16 \%$ & $103 / 7$ & $\begin{array}{c}0.65 \pm \\
0.06\end{array}$ \\
\hline & LC1404 & 132 & 360 & 32 & $0.02 \%$ & $\begin{array}{c}0.0005 \\
\%\end{array}$ & $0.0005 \%$ & $0.02 \%$ & $41 / 0$ & $\begin{array}{c}0.44 \pm \\
0.10\end{array}$ \\
\hline & LC1443 & 189 & 360 & 51 & $0.16 \%$ & $0 \%$ & $0.0001 \%$ & $0.16 \%$ & $99 / 9$ & $\begin{array}{c}0.56 \pm \\
0.07\end{array}$ \\
\hline
\end{tabular}

${ }^{1}$ Full sample names: LC0424, H03_072705_R0424; LC1408, 3881-1408; LC1404, 3869-1404; LC1443, 3869-1443.

${ }^{2}$ Unique sequences for $\mathrm{V} 6$ pyrosequencing tags were calculated after normalizing samples down to 14,869 total tags.

${ }^{3} \mathrm{Ti} / \mathrm{Tv}$ ratio is shown as numbers of transitions/transversions for clones and as decimal fraction for tags.

${ }^{4}$ Evenness derived from the Shannon-Weaver index and its standard deviation (calculated by DOTUR, Schloss 2005).

Table 1. Diversity comparison of the 16S rRNA, V6 hypervariable region, and ITS region among Lost City carbonate chimney samples. 

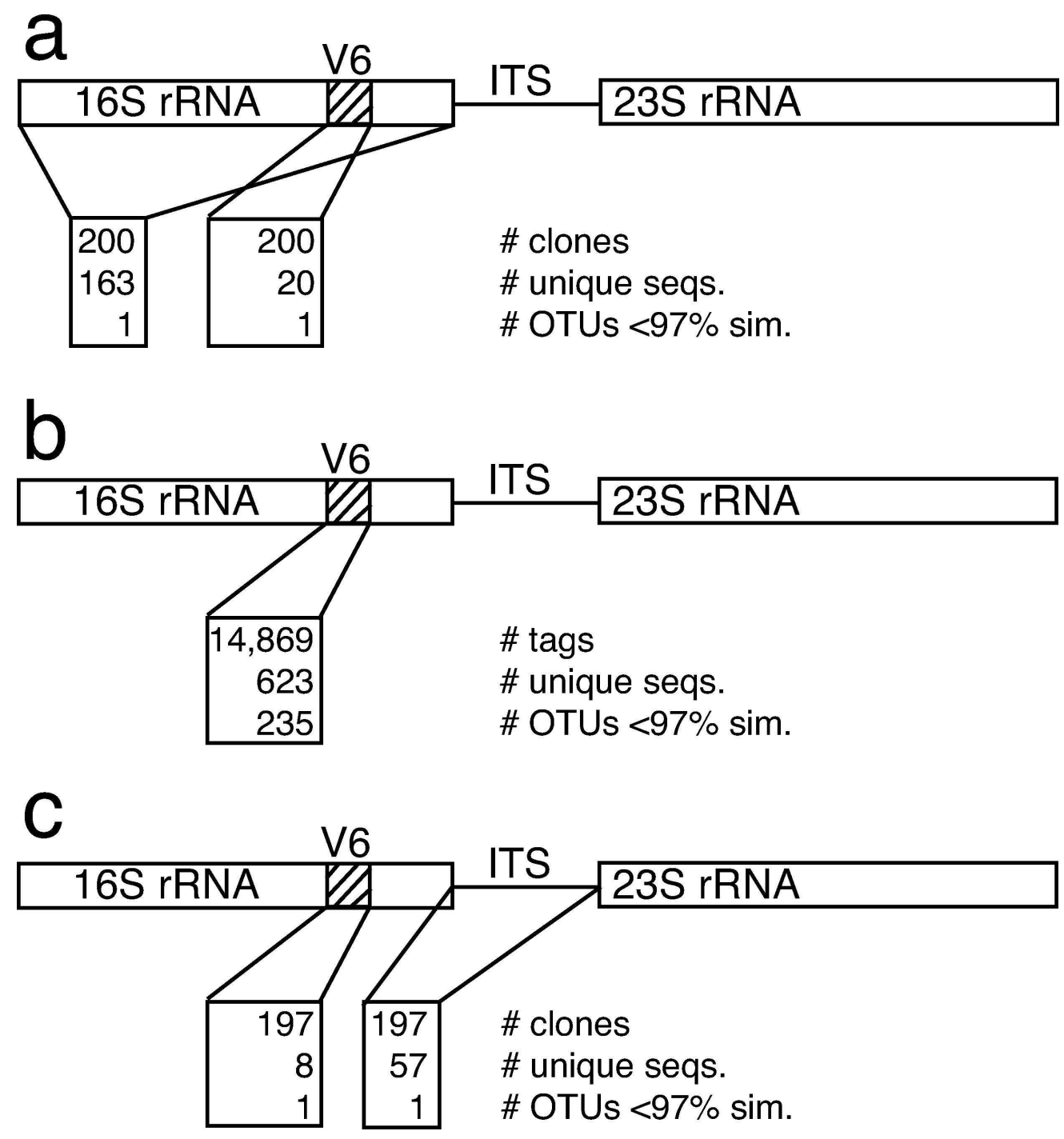

Comparison of tag pyrosequencing and clone library data from the same carbonate chimney. All sequences from 200 nearly full-length 16S rRNA clones obtained from sample LC0424 were more than $97 \%$ similar to each other (A). Collection of 14,869 tag pyrosequences of the V6 hypervariable region from a different sample (LC1408) of the same chimney revealed much greater diversity (B). A clone library constructed with DNA from sample LC1408 spanning the V6 hypervariable region and the intergenic transcribed spacer (ITS) region showed more diversity in the ITS region (C). $105 \times 120 \mathrm{~mm}(600 \times 600 \mathrm{DPI})$ 


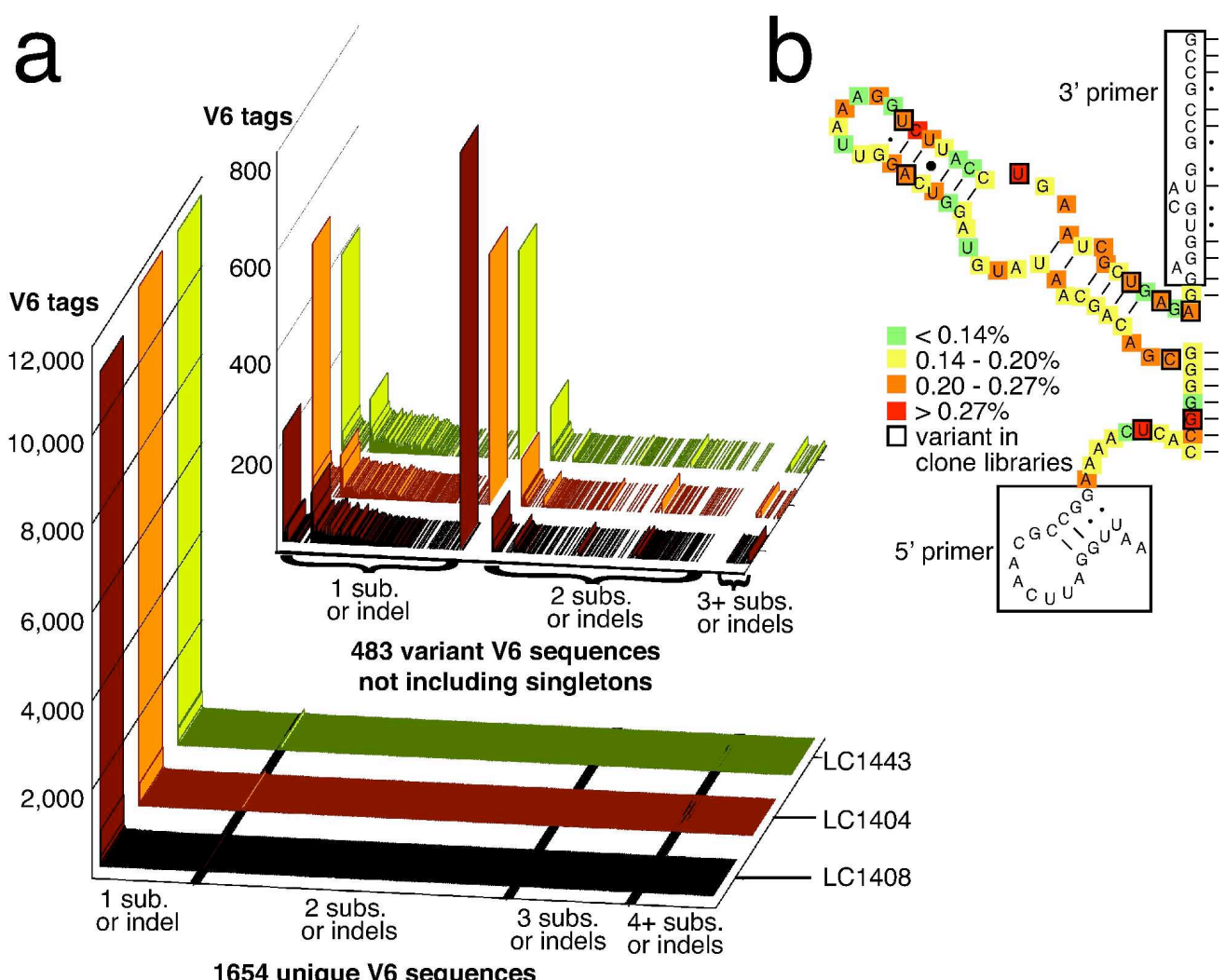

sorted by similarity to dominant sequence

Tag pyrosequences of the V6 hypervariable region reveal a wide range of highly similar, rare sequences. The relative abundance distribution (A) of 1654 different V6 sequences among the three samples (LC1408, LC1404, LC1133) shows the extreme dominance of one sequence and the diversity of rare sequences. Differences (and similarities) among samples are more easily seen when the one dominant sequence and sequences observed only once in the total dataset

('singletons') are omitted to show only the 483 most common variants (inset). Sequences are sorted along the $X$ axis by distance to the dominant sequence. The predicted secondary structure of the $\mathrm{V} 6$ region (B) was slightly modified from the archaeal structure on the Comparative RNA Web Site (http://www.rna.ccbb.utexas.edu) to fit the dominant sequence. Those bases that are variable in at least two clones among all the clone libraries in this study (in boxes) are among the most highly variable (orange and red shading) in the V6 tag dataset as well. All V6 sequence data is available at the VAMPS database, http://vamps.mbl.edu., under dataset name ICM_LCY_Av6 and in the NCBI Short Read Archive under submission number SRP000912. $167 \times 141 \mathrm{~mm}(600 \times 600 \mathrm{DPI})$ 

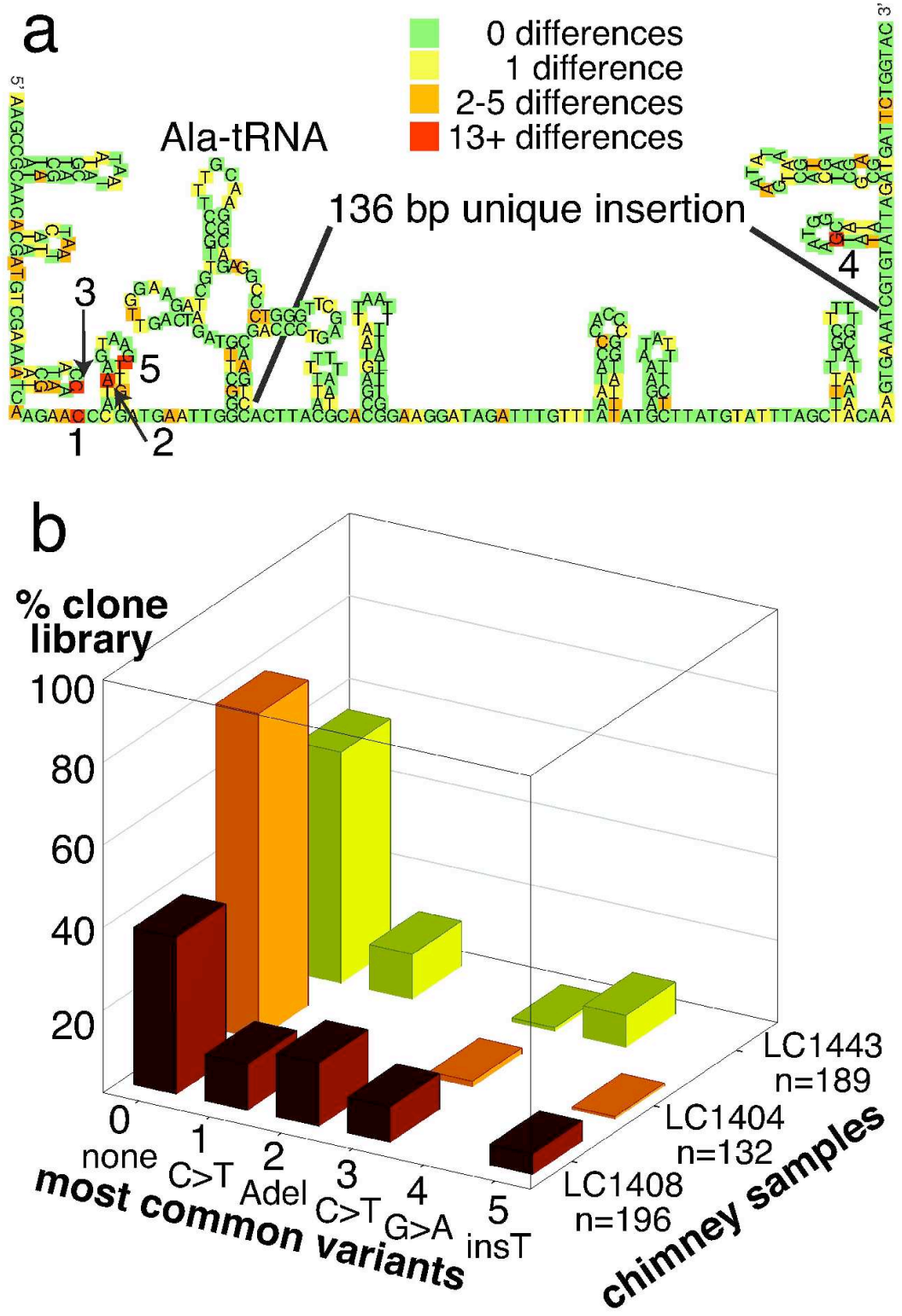

The variability of specific bases within the ITS region of Lost City Methanosarcinales differs among chimney samples. The secondary structure of the ITS (A) was predicted by UNAFOLD (Markham and Zuker, 2005) and modified to match the tRNA structure predicted by tRNAscan-SE (Lowe and Eddy, 1997). Bases are color-coded to indicate the number of clones (out of 517 total) that differed from the dominant sequence at that position, and the five most variable positions are numbered and compared among samples in (B). The most frequent variation, a C to T transition, occurred in 22 clones in sample LC1408, in 0 clones in LC1404, and 21 clones in LC1443. Accession numbers for clones including the V6 and ITS regions include GQ272945-GQ273460.

$$
109 \times 163 \mathrm{~mm}(600 \times 600 \mathrm{DPI})
$$




\section{Supplementary Information}

Multiple scales of diversification within natural populations of archaea in hydrothermal chimney biofilms

William J. Brazelton $^{1 *}$, Mitchell L. Sogin ${ }^{2}$, and John A. Baross ${ }^{1}$

${ }^{1}$ School of Oceanography and Center for Astrobiology and Early Evolution, University of Washington

2 Josephine Bay Paul Center, Marine Biological Laboratory at Woods Hole

\section{Sample descriptions}

Carbonate chimney samples LC1408 (full sample name 3881-1408), LC1404 (3869-1404), and LC1443 (3869-1443) were collected from the Lost City Hydrothermal Field (LCHF, depth, 735 m; latitude, 30.12; longitude, -42.12) with DSV Alvin during cruise AT07-34 aboard the R/V Atlantis in April/May 2003 (http://www.lostcity.washington.edu). Sample LC0424 (H03_072705_R0424) was collected by DSV Hercules during the 2005 Lost City Expedition aboard the R/V Ronald H. Brown. LC0424 and LC1408 were collected from a site known as Marker 3 or 'Poseidon,' a 60 m tall edifice emitting fluids at temperatures ranging from $55-88^{\circ} \mathrm{C}$ (Kelley et al. 2005). LC1408 minerals appeared bright white in color, very friable, and not lithified. Samples LC1404 and LC1443 are from a structure named Marker $\mathrm{C}, \mathrm{a} \sim 50 \mathrm{~cm}$ wide flange structure with several small (centimeters tall) chimneys growing on the top of the flange. LC1404 was collected from the front of the flange, and LC1443 was a small spire collected from the top. Both samples were cream white with a reddish discoloration that remains unexplained (Ludwig et al., 2006). Additional published characteristics of the samples are summarized in Table S1.

Shipboard, subsamples of chimney material were frozen immediately at $-80^{\circ} \mathrm{C}$ and remained frozen until onshore analysis. DNA was extracted from carbonate chimney samples according to a protocol 
modified from previous reports (Brazelton et al., 2006; Barton et al. 2006) and summarized here. After crushing a frozen carbonate sample with a sterile mortar and pestle, approximately $0.25-0.5 \mathrm{~g}$ of chimney material were placed in a $2 \mathrm{~mL}$ microcentrifuge tube containing $250 \mu \mathrm{L}$ of $2 \mathrm{x}$ buffer AE $(200$ mM Tris, $50 \mathrm{mM}$ EDTA, $300 \mathrm{mM}$ EGTA, $200 \mathrm{mM} \mathrm{NaCl,pH} \mathrm{8)} \mathrm{and} 2 \mu \mathrm{g}$ of poly-dIdC (SigmaAldrich) and incubated at $4^{\circ} \mathrm{C}$ overnight to allow chelation of salts and binding of DNA to poly-dIdC. Between 36-72 replicate tubes were processed in parallel, and approximately $15 \mathrm{~g}$ of carbonate minerals were processed for each sample. Proteinase K (final concentration $1.2 \mathrm{mg} / \mathrm{mL}$ ) and $10 \mu \mathrm{L}$ of $20 \% \mathrm{SDS}$ were added to each tube before incubation at $37^{\circ} \mathrm{C}$ for at most $30 \mathrm{~min}$. A further $150 \mu \mathrm{L}$ of $20 \%$ SDS and $500 \mu \mathrm{L}$ of phenol:chloroform:isoamyl alcohol (25:24:1 ratio by volume) were added to each tube before centrifugation at $12,000 \mathrm{~g}$ for $10 \mathrm{~min}$. Supernatants were transferred to clean tubes for a second phenol:choloroform:isoamyl alcohol extraction. After centrifugation, supernatants were pooled into SnakeSkin dialysis tubing (Pierce) and dialyzed against $20 \mathrm{mM}$ EGTA overnight at $4^{\circ} \mathrm{C}$. This large scale dialysis step proved to be very efficient in removing inorganic minerals and organic inhibitors. After dialysis, DNA was precipitated by adding 0.1 vol $3 \mathrm{M}$ sodium acetate and 1 vol isopropanol and stored at $-20^{\circ} \mathrm{C}$ for $2-4$ hours. Pellets were collected by centrifugation at $16,000 \mathrm{~g}$ for $20 \mathrm{~min}$ at $8^{\circ} \mathrm{C}$, washed once in $70 \%$ ethanol, dried in a vacuum centrifuge, and resuspended in TE (10 mM Tris, 1mM EDTA, $\mathrm{pH} 8$ ). Typical yield was $\sim 35 \mathrm{mg}$ of DNA per $\mathrm{g}$ of carbonate chimney material.

\section{Construction and sequencing of clone libraries}

Two 16S rRNA clone libraries including a total of 486 clones (GenBank accession numbers FJ791302FJ791787) from sample LC0424 were constructed by the DOE Joint Genome Institute according to the standard protocol published on their website: http://my.jgi.doe.gov/general/index.html. The V6-ITS clone libraries including a total of 516 clones from three samples (accession numbers GQ272945GQ273460) were constructed from amplicons covering the 16S rRNA V6 region downstream through the intergenic transcribed spacer (ITS) region to the $23 \mathrm{~S}$ rRNA. PCR amplification was conducted 
according to the protocol of Huber et al. (2006). The forward primer (886F-LCMS:

GAAGTACGGCCGCAAGGC) targets a region just upstream of the Lost City Methanosarcinales V6 region, and the reverse primer (58Ra: GCTTATCGCAGCTTGSCACG) targets the 5' end of the archaeal 23S rRNA gene (Huber et al. 2006). V6-ITS amplicons were reconditioned using the protocol of Thompson et al. (2002) and cloned using the TOPO-TA cloning kit (Invitrogen) according to the manufacturer's instructions. Cloned inserts were sequenced at the University of Washington HighThroughput Genomics Unit (www.htseq.org) with sequencing primers described by Huber et al. (2006). Because of inhibitors that could not be removed from the DNA preparations, PCR amplification of V6-ITS clones required 34-38 cycles of PCR amplification. It is possible that the higher evenness in LC1408 (Table 1) resulted from the higher number of cycles (38) used during PCR amplification of this sample compared to other two samples, which required only 34 cycles. The higher diversity in LC1408 and LC1443 compared to LC1404, however, is unlikely to be affected by cycle number or polymerase error, because only 34 cycles were used for both LC1443 and LC1404 and because of the high mutation rates in these libraries compared to that expected from polymerase and sequencing error, as described in the main text. More amplification cycles may have been required for sample LC1408 because it contained 100x lower archaeal density than the other two samples (Table X?) even though efforts were made to equalize DNA template concentrations. All alignments were calculated with MUSCLE (Edgar et al., 2004).

\section{Analysis of tag pyrosequences}

Protocols for construction and sequencing of V6 amplicon libraries have been described previously (Sogin et al., 2006; Huber et al., 2007). Tag sequences were screened for quality as recommend by Huse et al. (2007). Sequences assigned to the family Methanosarcinaceae by GAST (Huse et al., 2008) were aligned with MUSCLE (Edgar et al., 2004). Distance matrices were calculated with quickdist as described by Sogin et al. (2006) except that terminal gaps were penalized in our study because we 
inspected the 3' ends to confirm that primers were acurately trimmed and that the most common 3' deletions were not the result of incomplete sequences. Evenness values were derived from the Shannon-Weaver index as calculated by DOTUR (Schloss et al., 2005), and 97\% sequence similarity OTUs were calculated with DOTUR. To normalize relative abundances of each sequence among samples, tags were randomly resampled down to the sample with the fewest tags (LC1408: 14,869 tags) using Daisy-Chopper (available at http://www.genomics.ceh.ac.uk/GeneSwytch/Tools.html).

\section{Community similarities among samples}

The abundance distributions of tag sequences in the three samples were highly similar, though sample LC1404 is more similar to LC1443 (94\% Bray-Curtis similarity), which was sampled $20 \mathrm{~cm}$ away on the same chimney, than to LC1408 (90\% Bray-Curtis similarity), which was collected from a different chimney. After removing the one dominant sequence (because the Bray-Curtis index is weighted toward dominant members) and sequences occurring only once in one sample (to decrease the number of heavily undersampled sequences), the abundance distributions of the 483 remaining sequences (Fig. 2a) yielded a greater Bray-Curtis similarity between samples from the same chimney (LC1404 and LC1443, 79\%) than between samples from different chimneys, (70-71\%). If only very rare sequences (represented by fewer than 10 tags in each sample after normalization) were considered in the similarity calculation, the same trend was observed: LC1404 and LC1443 were $46 \%$ similar but only 35-38\% similar to LC1408 according to the Bray-Curtis index. Therefore, the abundances of dominant as well as rare sequences are more similar in samples from the same chimney than in samples from different chimneys.

\section{References}

Barton, H.A., Taylor, N.M., Lubbers, B.R., and Pemberton, A.C. (2006) DNA extraction from low- 
biomass carbonate rock: An improved method with reduced contamination and the low-biomass contaminant database. Journal of Microbiological Methods 66:21-31.

Bradley, A.S., Hayes, J.M., Summons, R.E. (2009) Extraordinary ${ }^{13}$ C enrichment of diether lipids at the Lost City Hydrothermal Field indicates a carbon-limited ecosystem. Geochim. Cosmochim. Acta 73:102-118.

Brazelton, W.J., Schrenk, M.O., Kelley, D.S., and Baross, J.A. (2006) Methane- and sulfurmetabolizing microbial communities dominate the Lost City hydrothermal field ecosystem. Appl Environ Microbiol 72: 6257-6270.

Edgar, R.C. (2004) MUSCLE: multiple sequence alignment with high accuracy and high throughput. Nucleic Acids Res 32:1792-7.

Huber, J.A., Butterfield, D.A., and Baross, J.A. (2006) Diversity and distribution of subseafloor Thermococcales populations in diffuse hydrothermal vents at an active deep-sea volcano in the northeast Pacific Ocean. J Geophys Res Biogeosciences 111: G04016.

Huber, J.A., Welch, D.B.M., Morrison, H.G., Huse, S.M., Neal, P.R., Butterfield, D.A., and Sogin, M.L. (2007) Microbial population structures in the deep marine biosphere. Science 5:97-100.

Huse, S.M., Huber, J.A., Morrison, H.G., Sogin, M.L., and Welch, D.M. (2007) Accuracy and quality of massively parallel DNA pyrosequencing. Genome Biol. 8:R143.

Huse, S.M., Huber, J.A., Morrison, H.G., Sogin, M.L., and Welch, D.M. (2008) Exploring microbial diversity and taxonomy using SSU rRNA hypervariable tag sequencing. PLoS Genet 4:e1000255.

Kelley, D.S., Karson, J.A., Fruh-Green, G.L., Yoerger, D.R., Shank, T.M., Butterfield, D.A. et al. (2005) A serpentinite-hosted ecosystem: the Lost City hydrothermal field. Science 307: 14281434.

Ludwig, K.A., Kelley, D.S., Butterfield, D.A., Nelson, B.K., Fruh-Green, G. (2006) Formation and 
evolution of carbonate chimneys at the Lost City Hydrothermal Field. Geochim Cosmochim Acta 70:3625-3645.

Proskurowski, G., Lilley, M.D., Kelley, D.S., Olson, E.J. (2006) Low temperature volatile production at the Lost City Hydrothermal Field, evidence from a hydrogen stable isotope geothermometer. Chem Geol 229:331-343.

Proskurowski, G. et al. (2008) Abiogenic hydrocarbon production at lost city hydrothermal field. Science 319:604-7.

Schloss, P.D. and Handelsman, J. (2005) Introducing DOTUR, a computer program for defining operational taxonomic units and estimating species richness. Appl Environ Microbiol 71:15016.

Schrenk, M.O., Kelley, D.S., Bolton, S.A., Baross, J.A. (2004) Low archaeal diversity linked to subseafloor geochemical processes at the Lost City Hydrothermal Field, Mid-Atlantic Ridge. Environ Microbiol 6:1086-1095.

Sogin, M.L., Morrison, H.G., Huber, J.A., Welch, D.M., Huse, S.M., Neal, P.R., Arrieta, J.M., and Herndl, G.J. (2006) Microbial diversity in the deep sea and the underexplored "rare biosphere". Proc Natl Acad Sci USA 103:12115-20.

Thompson, J. R., Marcelino, L. A., and Polz, M.F. (2002) Heteroduplexes in mixed-template amplifications: formation, consequence and elimination by 'reconditioning PCR'. Nucleic Acids Res 30:2083-2088. 


\begin{tabular}{|c|l|c|c|c|c|c|c|c|c|c|}
\hline $\begin{array}{c}\text { Chimney } \\
\text { Sample }\end{array}$ & $\begin{array}{l}\text { Lhimney } \\
\text { Location }\end{array}$ & $\begin{array}{c}\text { Max } \\
\text { fluid } \\
\text { temp } \\
\left({ }^{\circ} \mathrm{C}\right)\end{array}$ & $\begin{array}{c}\text { Max } \\
\text { fluid } \\
\mathrm{H}_{2} \\
\left(\mathrm{mmol}^{-1}\right) \\
\mathrm{kg}^{-1}\end{array}$ & $\begin{array}{c}\text { Max } \\
\text { fluid } \\
\mathrm{CH}_{4} \\
\left(\mathrm{mmol}_{\left.\mathrm{kg}^{-1}\right)}\right.\end{array}$ & $\begin{array}{c}\text { Cells g } \\
\text { dry } \\
\text { weight }\end{array}$ & Archaea $^{\mathrm{a}}$ & Bacteria $^{\mathrm{b}}$ & LCMS $^{\mathrm{b}}$ & $\begin{array}{c}\text { Total } \\
\text { organic } \\
\text { carbon } \\
(\%)\end{array}$ & $\begin{array}{c}{ }^{\mathrm{TM}^{13}} \mathrm{C}_{\text {toc }}(\% \circ \\
\text { vs. VPDB })\end{array}$ \\
\hline LC1408 & Marker 3 & 88 & 13.26 & 1.55 & $2.0 \times 10^{-8}$ & $25 \%$ & $14 \%$ & $18 \%$ & n.d. & n.d. \\
\hline LC1404 & Marker C & 70 & 14.38 & 1.98 & $\begin{array}{c}1200 \times 10^{-} \\
8\end{array}$ & $41 \%$ & $8 \%$ & $32 \%$ & 0.20 & -7.8 \\
\hline LC1443 & Marker C & 70 & 14.38 & 1.98 & $\begin{array}{c}1600 \times 10^{-} \\
8\end{array}$ & $38 \%$ & $10 \%$ & $21 \%$ & n.d. & n.d. \\
\hline
\end{tabular}

${ }^{a}$ Determined by DAPI-staining

${ }^{\mathrm{b}}$ Percentage of DAPI-stained cells detected by FISH probe specific to each group

Table S1. Previously published characteristics of the three carbonate chimney samples from which V6 tags and V6-ITS clone libraries were sequenced. Fluid temperatures and concentrations of $\mathrm{H}_{2}$ and $\mathrm{CH}_{4}$ are maximum values reported by Proskurowski et al. (2006 \& 2008). Cell densities and proportions of phylogenetic groups are from Schrenk et al. (2004) and M. Schrenk (doctoral dissertation, 2005). Organic carbon concentrations and isotopic measurements are from Bradley et al. (2009). Fluid temperature and chemistry are identical for samples LC1404 and LC1443 because these carbonate samples were collected from the same chimney. 


\section{Figure S1 with caption below:}

thermoauto_A thermoauto_B thermophila_A thermophila_B
LCMS

stadtmanae_A stadtmanae_B stadtmanae_C stadtmanae_D burtonii_A burtonii_B burtonii_C barkeriA barkeriB barkeric mazeiA mazeiB acetivoransB acetivoransB
acetivoransA acetivoransA

101

thermoauto_A thermophila thermophila_B LCMS

stadtmanae_A stadtmanae_B stadtmanae_C stadtmanae_D burtonii_A burtonii_B burtonii_c barkeriA barkeriB mazeiA mazeiB mazeic acetivoransB acetivoransA acetivoransC

201

thermoauto_A thermoauto_B thermophila_A LCMS

stadtmanae_A stadtmanae_B stadtmanae_C stadtmanae_D burtonii_A burtonii_B burtonii_C barkeriA barkeriB barkeric mazeiA mazeiB acetivoransB acetivoransB acetivoransA

thermoauto_A thermoauto_B thermophila_A LCMS

stadtmanae_A stadtmanae_B stadtmanae_C stadtmanae_D burtonii_A burtonii_B burtonii_C barkeriA barkeri barkeric mazeif mazeiB mazeiB acetivoransB acetivoransB acetivoransA
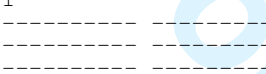
$-$

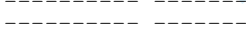

TCTGTATCCT

TCTGTATCCT

TTTGG

TTGGGG--

CTGTGGATCT CTAGTCTCTC

TTTGGATCTC TTGTCTCT--

TCCATAT-

TTATGGATCT CTCGTCTCTC

301

$--$
TACACAAA-- ------AAGA ATAAAG-TACACAAA -- ------AAGA ATAAAG--

\section{$-----------------------$} AATATAC-- ------ AAGT $-1,----1$ AATATAC--- ------ AATT AAAGA--AACATATAAA ATTTATAAGT AAAGGATAAT AACATATAAA TTTA--TAAT AAAGGATAAT AATTTCATA AACATATAAA TTTA--TAAT AAAGGATAAT AATTTTATAT

--------- ------ AAGC A-------
-AAGC A

-------------- AAGC A-------

-

------------ AAGC AAAA-

--- ------- AAGC ATAA-

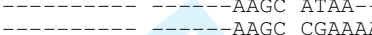

-

-AAGC CGAAAA--

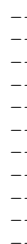

---
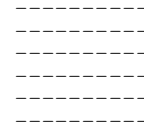

- - - - - -

---AGATGTG TGCTTTTCG- ---GGGATTA CTC---CTCC CACTG--TGA TGGGGC------AGATGTG TGCTTTTCG- ----GGGATTA CTC---CTCC CACTG--TGA TGGGGC--AGTGTG CATAA----- - -------A TCG---GCCG GAAGC--TGA TAGG---

---AGTGIG CATAA----- -------A TCG---GCCG GAAGC--TGA TAGG-----CGATCTG TATAATACAG ATCAACACTA CTA---ATTA GATGT--CGA AAAACTA--

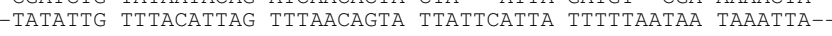
CCAAAATTTG TGTACA---- -- TACACTA TTA---TATG AATTT--TAA TAAGTTATTT $\begin{array}{llll}\text { CCAAAATTTG TGTACA---- } & --- \text { TACACTA } & \text { TTA---TATG } & \text { AATTT--TAA TAAGTTATTT } \\ \text { CC-AAATTTG TGTACA---- } & --- \text { TACACTA } & \text { TTA---TATG AATTT--TAA } & \text { TAAGTTATTT }\end{array}$ --AGATCCG CACAAAGCGG ATCACCGCTA TCA---GTCA GAAAT--CGA TAAACTG--- AGATCCG CACAAAGCGG ATCACCGCTA TCA---GTCA GAAAT--CGA TAAACTG---AgATCCG CACAAAGCG ATCACCGCTA TCA---GTCA GAAAT--CGA TAAACTG--

-

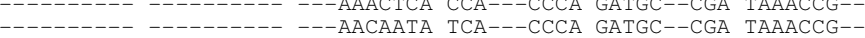
------- ------- -- - AACACTA CCA---CCCA GATGC--CGA TAAACCG--AACACTA TCA---CCCA GATGC--CGA TAAACCG---
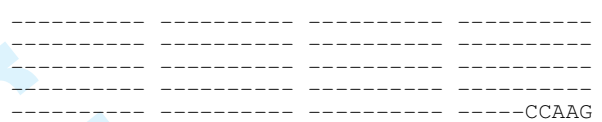
-

-ACCTTAACT GT------- TCTGGTTCTA ACCTTAACT GT--.---- TCTGGTTCTA TTCGTCACT TGACCTGTTG CTGGGATCTA TTCGTCACT TGACCTGTTG CTGGGATCTA TTTCATATTC ATCATATTTT ACAAACATGT AATTGTTTGT CTTATAGGTG CAGCTTACAT TTTAACAG-- -ATGATAAGT GAA------- --GAGATGAA TTTCATATTC ATCATATTTT ACAAACATGT AATTGTTTGT CTTATAGGTG CAGCTTACAT TTTAACAG-- -ATGATAAGT GAA------- --GAGATGAA TTTCATATTC ATCATATTT ACAAACATGT AATTGTTTGT CTTATAGGTG CAGCTTACAT TTTAACAG-- -ATGATAAGT GAA------- --GAGATGAA (1)

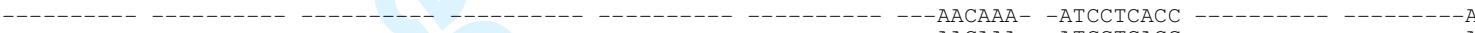

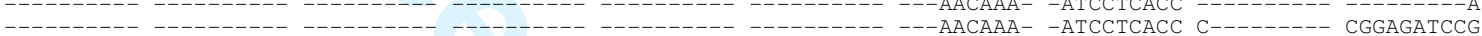
$\begin{array}{llll} & \end{array}$ -
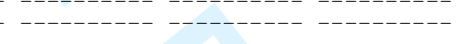

-ATCAA-- -ATCCTCACC

ATCCTCACC

ATTAAATGAT

ATtAAATATT

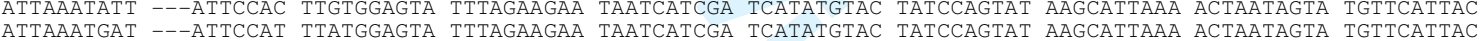
ATTAAATATT ---ATTCCAT TTATGGAGTA TTTAGAAGAA TAATCATCGA TCATATGTAC TATCCAGTAT AAGCATTAAA ACTAATAGTA TGTTCATTAC CTGGAAAGT CTGGAAAGTT -------------- CTCA GTTGGATCAA T-

TTTAAATCAT CGATCATAAT CTAATGATCA ATTCTAA---

TTTAAATCAT CGATCATAAT CTAATGATCA ATTCTAA--

-TttTtAatg GAttTtCCtt tgGtgCAgCC GC

-TTTTTAATG GATTTTCCTT TGGTGCAGCC GC

-

TGGCACTACC AAGCAGAGG CCA-TAGATT AGAAARAAAT AAGTCCATAC AC--AT-- TTTTTAATGC ACCGAGTAAT TAATTT--- - TGGCATAC IAACTAGAG- ----TAGATT AGAAAAAAAT AAGTCCATAC AA---TTT-- GTATTGATTT CTAATATTAT TAATTTATTC AATTAGTTTG TGGCACTAAC IAACTAGAG- ---TAGATTA GAAAAAAAAT AAGTCCATAC AA--TTTG-- TATTGATTTI TGAGIATTAT TAATTIAIC AATTGGTTTG

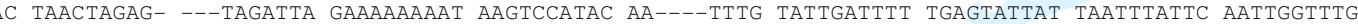

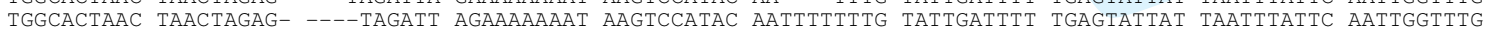
DAAGTGCACCG AGTAAGATI CACAATCATC AAGTGCACCG AG-- - CTCATC AAATGCACCC GG - - -

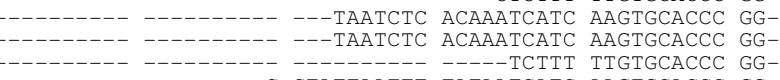

-

CTATAAATT

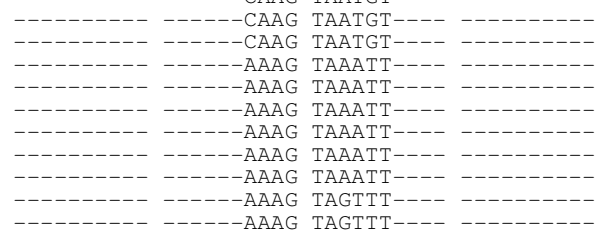


401

\begin{tabular}{|c|c|c|c|c|c|c|}
\hline hermoauto_A & GT & -- & GGT & ITG & & \\
\hline moauto_B & $--\mathrm{TGT}$ & GGG-- & ---ATGTGGT & GGTGAAGTTG & ATGATATG & \\
\hline mophila_A & -------- & --- & --- GGAGAAC & TGCTCCACAG & GGAAGGGCTG & \\
\hline mophila_B & --------- & ----------- & --- GGAGAAC & TGCTCCACAG & GGAAGGGCTG & \\
\hline MMS & --- TATTT & GGG-------- & --- AAGGAT & $\mathrm{A}--------$ & ---- GATTTG & TTT \\
\hline adtmanae_A & TTAGATGTTT & GTG-TAATAT & ATAATAGAAT & AGTAATATTT & CTATGGTTTA & CTTE \\
\hline adtmanae_B & TTAGATGTTT & GTGTAATAAT & ATAATAGAAT & AGTAATATTT & CTATGGTTTA & CTTAAATA \\
\hline nae_c & TTAGATGTTT & GTGTAATAAT & ATAATAGAAT & AGTAATTCT- & --ATGGTTTA & CTTAAATA \\
\hline ae_D & TTAGATGTTT & GTGTAATAAT & ATAATAGAAT & AGTAATTCT- & --ATGGTTTA & $\mathrm{CT}$ \\
\hline A & $-\ldots-$ TGCTT & GGG------- & $---\mathrm{AAGGAT}$ & $\mathrm{G}-------$ & --- GATGTG & CCTGA \\
\hline B & $----\mathrm{TGCTT}$ & GGG------- & ---- AAGGAT & G---------- & ---- GATGTG & \\
\hline $\mathrm{C}$ & $----\mathrm{TGCTT}$ & GGG-------- & $----\mathrm{AAGGAT}$ & G---------- & ---- GATGTG & $\mathrm{CCTGA}-$ \\
\hline & ---- TTC & GGG-------- & --- GAAGGGC & GGATTGCCTG & CGTTGACACG & \\
\hline iB & ----- TTC & GGG-------- & ---GAAGGGC & GGATTGCCTG & CGTT & \\
\hline & ----- TTC & GGG-------- & --- GAAGGGC & CTG & $\mathrm{ACG}$ & \\
\hline $\mathrm{z}$ & $------\mathrm{TTC}$ & GGG-------- & ---GAAGGAT & GGAT & ACCG & \\
\hline & ----- TTC & GGG-------- & $---G P$ & CTG & $\mathrm{CCG}$ & \\
\hline zeic & ------ TTC & GGG-------- & --- GAAGGAT & GGA & $\mathrm{CCC}$ & \\
\hline $\mathrm{sB}$ & ----- TTC & GGG-------- & $---G P$ & CTG & $\mathrm{GCT}$ & \\
\hline ISA & $-\ldots-$ TTC & GGG------- & ---GAAGGAT & GGATAGCCTG & TGCTGAAGCT & \\
\hline & & & & & & \\
\hline & & & & & & \\
\hline A & & $---------\mathrm{T}$ & $\mathrm{ACG}$ & 171 & & \\
\hline & --------- & $---------\mathrm{T}$ & $\mathrm{ACG}$ & $\mathrm{AA}$ & A & \\
\hline la_A & ---------- & --------- & $------\mathrm{AAT}$ & $\mathrm{AGG}$ & $\mathrm{CCA}-$ & \\
\hline la_B & ---------- & --------- & $-------\mathrm{AAT}$ & AGG & $\mathrm{CCA}-$ & \\
\hline & TGTATtTAGC & TTTTTTGCTT & TTGCATAAAA & AAA & TCG-- & \\
\hline A & TTTGC & TTT & AAA & & ATATTAT & TTT \\
\hline$B$ & ITTGC & TTTGT & AAA & $A G$ & TAT & \\
\hline C & TTTGC & TTT & AAA & $\mathrm{AG}$ & TAT & \\
\hline D & TATTTTTTGC & TTTGTTTGAT & AAA & AG & ACAATATTAT & TTT \\
\hline & --------- &.---- TACC & $\mathrm{AGG}$ & TA & TCA- & \\
\hline $\mathrm{B}$ & -------- & ------ TACC & AGG & $\mathrm{GA}$ & TCA- & \\
\hline & - - - - - - - & ------ TACC & CCATA & $\mathrm{GA}$ & TCA- & \\
\hline & --------- & ---------- & $------\mathrm{AGG}$ & $T A$ & TCA- & \\
\hline & --------- & 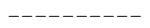 & $-------\mathrm{AGG}$ & $\mathrm{TA}$ & TCA- & \\
\hline & --------- & ---- & ------- AGG & $T A$ & TCA- & \\
\hline & -------- & ---- & $-------\mathrm{AGG}$ & $A G$ & TCG- & \\
\hline & $---1 \quad r y$ & --1 & $-------\mathrm{AGG}$ & $\mathrm{CA}$ & TCG- & \\
\hline & ---- & ---- & $-------\mathrm{AGG}$ & $\mathrm{CAC}$ & TCG- & \\
\hline $\mathrm{sB}$ & $-------\infty-$ & ------- & ------- AGG & $\mathrm{CA}$ & TCG- & \\
\hline & ---------- & ----------- & ------- AGG & CATA-TGAAG & TCG- & \\
\hline & & & & & & \\
\hline & & & & & & \\
\hline & & & & & & \\
\hline & & & - & & & \\
\hline $\bar{a} \_A$ & AGTGG & $\mathrm{GAC}$ & CTGC & & & \\
\hline a_B & & GAC & & & & \\
\hline & GTGA & GAGI & GGT & - & & \\
\hline & & & AAAT & TTT & & \\
\hline$B$ & GTGA & AATT & $\mathrm{AAT}$ & TTTT & & \\
\hline & $\mathrm{A} z$ & AAT & $\mathrm{AAT}$ & TTTT & & \\
\hline D & GTGA & AATT & AATAAAAAAT & TTTTT & & \\
\hline & & GATG & & & --- & \\
\hline i_B & GTGA & GATG & GGI & - & & \\
\hline i_c & GTGA & GATG & GGTA - & --- & ---------- & \\
\hline & GTTA & GGAT & GAA & & & \\
\hline & CT-GGTTA & GGAT & -- & ---1 & -- & \\
\hline & ACCT-GGTTA & GGATACACAG & GAA- & & & \\
\hline & CT-GGTTA & GGTA & & -- & -- & \\
\hline & CCT-GGTTA & GGTA & AAT---- & & & \\
\hline & CT-GGTTA & GGTA & $\mathrm{AA}--------$ & ----- & -- & \\
\hline & CT-GGTTA & GGT & -------- & -------- & --------- & --- \\
\hline iv & ACCT-GGTTA & GGTAATTAGG & AATTATGCTA & TCAGGTGGAT & GGCTCGGCTC & AAGAGCTT \\
\hline SI & $A G 1-A G 1$ & & & & & \\
\hline
\end{tabular}

GATTGTAATC --CAAAACTG GCATTAACTG ACCAGAgAGA GATTGTAATC --CAAAACTG GCATTAACTG ACCAGAGAGA GTATACGCTT TGCAGACCAG ACGCTCACTG ------- A C GTATACGCTT TGCAGACCAG ACGCTCACTG T GTATATGAAT GGCATATTAG ACGCTCACTG $-\cdots-----$ A

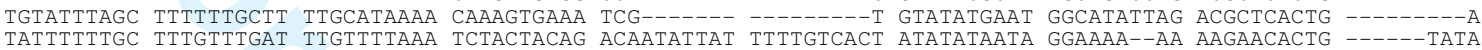
TATTTTTGC TTTGTTTGAT TTGITTAAA TCIACTACAG ACAATATIA TITGICACI AIATATAATA GGAAAA--AA AAGAACACTG ------IATA TATTE TATC (1) GTATACATAT TACATATCAG ACGCTCACTG --------G GTATACATAT TACATATCA ACGCTCACTG $---------G$ GTATACATAT TACATATCAG ACGCTCACTG $---------G$ T GTATAAGTGC TGTATACTGG ACGCTTTCTG --------- G GTATAAGTGC TGTATACTGG ACGCTTTCTG --------- G T GTATAAGTGC TGTATACTGG ACGCTTTCTG ---------- G GTATAGGTGC TGTATATTGA ACGCTAACTG --------- G $\begin{array}{llll}\text { GTATAGGTGC } & \text { TGTATATTGA } & \text { ACGCTAACTG } & ---------G \\ \text { GTATAGGTGC } & \text { TGTATATTGA ACGCTAACTG } & ---------G \\ \text { GTATATGTGC } & \text { TGTATACTGG } & \text { ACGCTTACTG } & --------\end{array}$ GTATATGTGC TGTATACTGG ACGCTTACTG TATATGIGC TGTATACIGG ACGCTTACTG ---------G

Figure S1. The LCMS ITS region encodes a tRNA and shows sequence similarity to the ITS regions of several methanogens. The alignment includes sequences from: Methanosaeta thermophila (NC_008553), Methanosarcina barkeri (NC_007349), Ms. acetivorans (NC_003552), Ms. mazei (NC_003901), Methanococcoides burtonii (NC_007955), Methanobacterium thermoautotrophicus (NC_000916), Methanosphaera stadtmanae (NC_007681), and Lost City Methanosarcinales (GQ273207). 


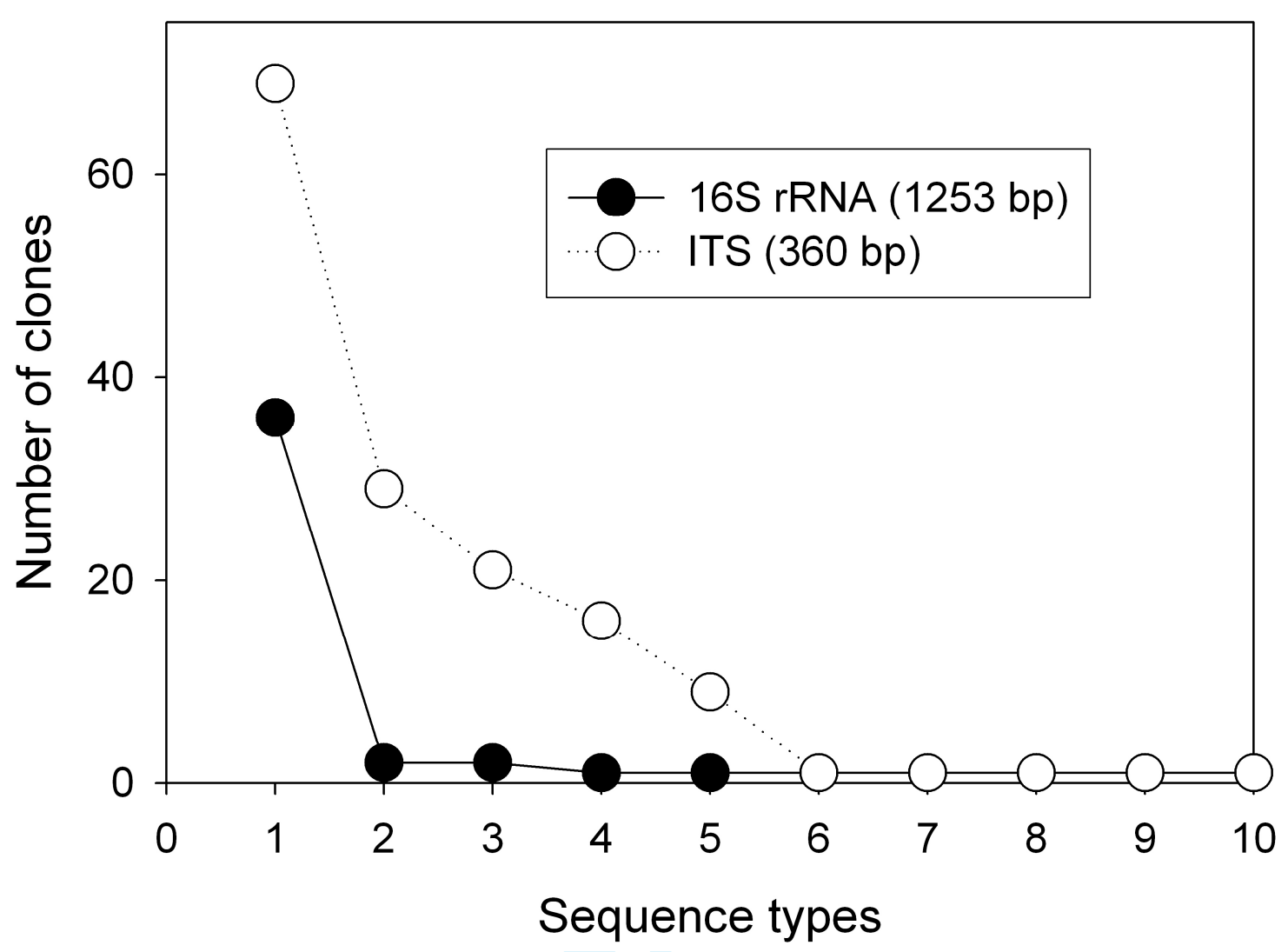

Figure S2. Rank-abundance plot showing the number of clones sharing the 10 most frequently occurring 16S rRNA and ITS sequences in samples LC0424 and LC1408, both of which were collected from the Poseidon chimney (Marker 3). Only one 16S rRNA sequence occurs more than twice, but five ITS sequences occur many times in this sample. As shown in Figure 3b, other samples contain different abundant ITS sequences. 